\title{
Current Situation and Analysis of the research on MOOC copyright
}

\author{
Xiangpeng Pan ${ }^{1, a,{ }^{*}}$, Fengtao Xiao ${ }^{1, b}$, Luobei Kuang ${ }^{1, c}$, Ruotong Liao ${ }^{1, d}$ \\ ${ }^{1}$ Military Continuing Education Technology Service Center of National University of Defense \\ Technology, Changsha, China
}

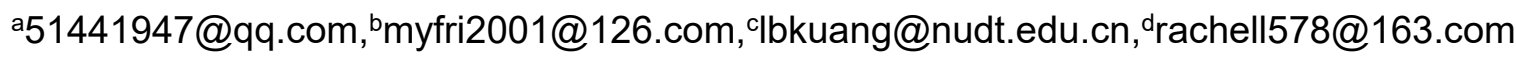

${ }^{*}$ Corresponding author

Keywords: Copyright, MOOC, Fair use.

\begin{abstract}
Widespread popularity of MOOC triggers people's high attention on its copyright. This thesis, based on analyzing domestic literature on MOOC copyright studies, mainly summarizes the availability of MOOC copyright, main contents covered, relevant stakeholders, protection methods of MOOC copyright as well as required support services and responsibilities on the library. Meanwhile, this thesis also provides some suggestions on construction and perfection of MOOC copyright.
\end{abstract}

\author{
MOOC 版权研究现状与分析 \\ 潘湘鹏 $1, a,{ }^{*}$ ，肖枫涛 ${ }^{1, b}$ ，匡罗贝 ${ }^{1, c}$, 廖若粀 $1, d$ \\ 1国防科技大学军事职业教育技术服务中心，长沙，湖南，中国
}

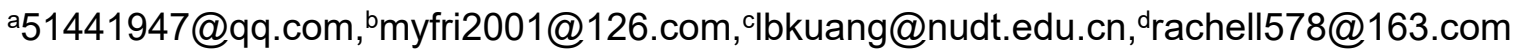

*通讯作者

关键词：版权；MOOC; 合理使用

摘要: MOOC的广泛兴起带来各方对有关版权问题的高度关注。本文通过梳理国内M00C版权研 究文献, 重点就M00C的可版权性、涵盖内容、利益相关者、保护形式和图书馆承担M00C版权 支持服务进行了归纳分析，并对版权建设与完善提出了建议。

\section{1. 引言}

MOOC（Massive Open Online Courses），即大规模在线开放课程，是指在计算机互联网 远程教育的基础上, 通过平台运营, 免费向公众开放的一种教育资源的开放获取模式和教育 模式。 ${ }^{[1]} 2013$ 年始, 国内一批优秀的 MOOC 平台陆续上线, 一批 MOOC 课程陆续建成, 据 教育部数据, 截至 2018 年 1 月, 有关高校和机构自主建成 10 余个国内慕课平台, 460 余所 高校建设 3200 多门 MOOC 上线, 累计有 5500 万人次高校学生和社会学习者选修慕课, 我国 的慕课数量现在已经稳居世界第一。 ${ }^{[2]} \mathrm{MOOC}$ 给众多学习者提供了更丰富的学习资源、更多 免费的学习机会和更简便的学习方法。

与此同时，MOOC 版权、MOOC 版权 (著作权) 的保护、MOOC 课程的合理使用等问 题, 成为 MOOC 发展的过程中急需解决的重点问题。本文主要梳理了国内 MOOC 版权研究 有关现状，对 MOOC 版权研究的内容进行对比分析和归纳总结，并提出了相关建议。 


\section{2. 国内MOOC版权研究的现状}

截止 2018 年 7 月, 在中国知网对 MOOC 版权文献进行检索, 以“主题=MOOC 版权 or 主 题=慕课版权”, 检索到论文 135 篇, 对这 135 篇论文进一步整理和筛选, 剔除不相关或大部 分不相关的文献, 得到有关 MOOC 版权相关的文献约 42 份, 从发表年份来看, 国内对 MOOC 版权的研究始于 2014 年, 从数量上看, 研究集中在 2015、2016 年, 文献数量分别为 11 篇、 14 篇。

表 1 是各年份 MOOC 版权研究研究层次分布情况, 从层次分布搜索结果来看, 国内 MOOC 版权研究起步比较晚, 研究数量是非常少, 大部分集中在基础研究的层次。

表1 2013年-2018年7月各年份MOOC版权研究层次分布情况

\begin{tabular}{|l|c|c|c|c|c|c|c|}
\hline $\begin{array}{l}\text { 研究年份 } \\
\text { 究层次 }\end{array}$ & 2013年 & 2014年 & 2015年 & 2016年 & 2017年 & $\begin{array}{c}\text { 2018年 } \\
\text { 7月 }\end{array}$ & $\begin{array}{c}\text { 小计 } \\
\text { (篇) }\end{array}$ \\
\hline 基础研究(社科) & 0 & 2 & 9 & 10 & 8 & 3 & 32 \\
\hline 行业指导(社科) & 0 & 2 & 2 & 3 & 0 & 0 & 7 \\
\hline 政策研究(社科) & 0 & 0 & 0 & 1 & 1 & 0 & 2 \\
\hline 国际法 & 0 & 1 & 0 & 0 & 0 & 0 & 1 \\
\hline 合 计 & 0 & 5 & 11 & 14 & 9 & 3 & 42 \\
\hline
\end{tabular}

表 2 是对以上发表的 42 篇文献的研究内容进行更细化的梳理。从表 2 中可以看出, 国内 对 MOOC 版权的研究内容研究主要集中在图书馆参与 MOOC 版权的实践、MOOC 版权保护 和 MOOC 合理使用, 研究内容相对集中, 说明这三个方面是需要不断探索和完善的, 2017 年后研究文献数量减少, 也说明 2017 年后有关问题已经初步形成共识, 结合表 1 可以看出, 2017 年后版权政策制定方面的研究开始出现, 希望版权解决方案固化成制度规范的需求进一 步增强; 各研究分类中, 对 MOOC 可版权特征的研究文献极少, 说明对 MOOC 可版权性各 方已形成共识。

表2 2013年一2018年7月MOOC版权研究内容的分布情况

\begin{tabular}{|l|c|c|c|c|c|c|c|}
\hline $\begin{array}{l}\text { 研究年份 } \\
\text { 研究内容 }\end{array}$ & 2013年 & 2014年 & 2015年 & 2016年 & 2017年 & $\begin{array}{c}\text { 2018年 } \\
\text { 7月 }\end{array}$ & $\begin{array}{c}\text { 小计 } \\
\text { (篇) }\end{array}$ \\
\hline MOOC可版权特征 & 0 & 1 & 0 & 0 & 1 & 0 & 2 \\
\hline MOOC平台著作权 & 0 & 1 & 0 & 0 & 0 & 0 & 1 \\
\hline MOOC合理使用 & 0 & 0 & 1 & 2 & 1 & 0 & 4 \\
\hline $\begin{array}{l}\text { 图书馆参与MOOC } \\
\text { 版权的实践 }\end{array}$ & 0 & 2 & 8 & 9 & 3 & 2 & 23 \\
\hline MOOC版权保护 & 0 & 1 & 1 & 2 & 3 & 1 & 8 \\
\hline MOOC版权归属 & 0 & 0 & 1 & 0 & 1 & 0 & 2 \\
\hline 其他 & 0 & 0 & 0 & 1 & 0 & 0 & 2 \\
\hline 合 & 0 & 5 & 11 & 14 & 9 & 3 & 42 \\
\hline
\end{tabular}

\section{3. 国内 MOOC 版权研究的主要内容分析}

依据表1中关于MOOC版权研究的42篇文献进行研究, 从各国法律规定着手分析MOOC 的可版权性, MOOC版权的涵盖内容, MOOC版权涉及的相关者, 同时对国内MOOC版权的 保护方式和图书馆承担MOOC版权支持服务的研究进行了综合分析。 


\subsection{MOOC 可版权性法律规定分析}

我国《著作权法实施条例》第四条著作权法和本条例中“下列作品”的含义第二款规定: “口 述作品, 是指即兴的演说、授课、法庭辩论等以口头语言形式表现的作品。”[3]明确规定了教 师授课属于《著作权法》保护的范畴。MOOC是一种在线课程, 其由教师创作并进行讲授, 由制作团队录制, 由院校或MOOC合作平台提供者提供给平台, 平台上线后供学习者使用, 这也是授课的一种方式, 应纳入《著作权法实施条例》的保护。而且MOOC是教师或制作团 队创作，是不可复制性，符合独创性的要求。

《保护文学艺术作品伯尔尼公约》是1886年制订，是世界上第一部国际版权公约，我国 于1992年加入该公约。公约第二条将“讲课、演讲、讲道和其他同类性质作品”列为“文学世术 作品”，作为保护对象。 ${ }^{[4]} \mathrm{MOOC}$ 是一种在线课程, 由教师讲授, 属于讲课, 受到版权保护。

《美国著作权法》第102条“著作权的客体”强调作品须有某种固定的有形特质载体才予以 保护。 ${ }^{[5]} \mathrm{MOOC}$ 将讲课形式以计算机二进制模式固定在MOOC平台上, 满足版权保护客体要 求, 受到版权保护。

综合我国及国外部分国家的法律规定，MOOC是版权意义上的“作品”，是具有可版权性， 在世界范围内应受到版权保护。

\subsection{MOOC版权的涵盖内容}

MOOC 是由教师开发、全球学习者都可以参与的在线课程, MOOC 的版权涵盖内容主要 包括五个方面: 一是教师在开发和制作课程时需要的所有素材, 包括文字、图片、音频、短 视频、口述内容、课后习题等材料的版权; 二是教师自己出版的教材、教师推荐的教材和其 他阅读材料的版权; 三是学习者在讨论区发布的内容和其作业成果的版权; 四是学习者在 MOOC 平台学习行为数据的版权; 五是制作完成后的 MOOC 成品视频的版权等。

\subsection{MOOC 版权利益相关者分析}

MOOC 因其制作及运营过程中涉及的内容非常广，其内容涉及到的利益相关者比较多， 有学校、授课教师、MOOC 的制作人员、参与 MOOC 学习的学习者、MOOC 平台提供者、 教材或其它辅助教材的出版商等多个利益相关者, 如图 1 所示, 各个利益相关者参与 MOOC 的动机和需求不同, 因而 MOOC 版权涉及相关者会非常复杂, 因此 MOOC 版权一般需要经 过学校、授课教师、MOOC 平台提供者和出版商之间的讨论, 取得共识后进行协议商订后才 能确定。[6]

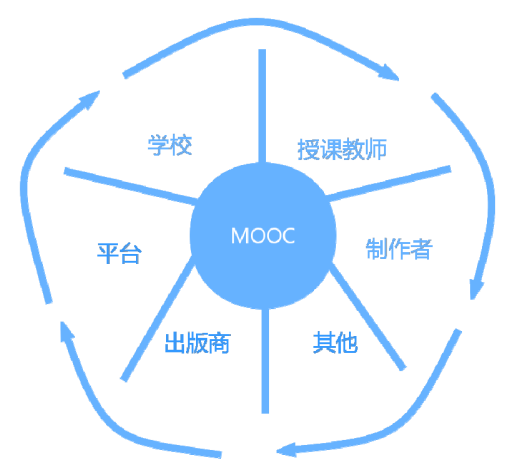

图1 MOOC版权利益相关者关系图

\subsection{MOOC 版权的保护形式及版权归属权利人的对比分析}

国内研究 MOOC 版权的保护形式主要有四种，第一形式是“协议形式约定各方权利”，来 源于《教育部关于国家精品开放课程建设的实施意见》第二部分第五项指出: “重视国家精品 开放课程的知识产权保护。在国家现行著作权法等知识产权法框架下, 以协议形式明确课程 
建设各方的权利、义务和法律责任，以保证国家精品开放课程持续建设和共享。”[7]第二种形 式是“版权集体管理”，来源于《著作权集体管理条例》第四章节“著作权集体管理活动”规定， 权利人与著作权集体管理组织订立著作权集体管理合同后, 著作权集体管理组织可以以自己 的名义主张权利, 可以与使用者订立许可使用合同、向使用者收取使用费, 转付给权利人, 并可以作为当事人进行有关权利的诉讼、仲裁活动。[8]第三种形式是“知识共享协议”, 来源 于《知识共享协议 ( CC ) 》规定, 著作权人允许使用者复制、散布、展示及演出其作品, 必 须保留其署名权。[9]第四种是“实名共享举报”，是指通过实名注册、共享监督、举报公布的 方式来确保版权归属。[10]

表 3 是对这四种形式从合规性、开放共享性、时间和经济成本、普遍性的对比分析，协 议形式约定各方权利是目前普遍采取的一种形式; 委托版权集体管理组织来解决 MOOC 建设 和管理中的版权问题, 这种方式的效率高, 时间成本和经济成本较低, 美国版权结算中心 (CCC)、德国多媒体结算中心(CMMV)等版权集体管理组织都在从事 MOOC 的授权申请、版 权清理等工作, 目前, 我国版权集体管理组织的种类较少, 还不能成为 MOOC 授权的主流渠 道; ${ }^{[11]}$ 知识共享协议的开放共享性最高, 但和实名共享举报的形式一样, 目前国内极少采用 这两种方式。

表3２013年一2018年MOOC版权保护四种形式对比分析

\begin{tabular}{|l|c|c|c|c|}
\hline 主要优点 & 协议形式约定各方权利 & 版权集体管理 & 知识共享协议 & 实名共享举报 \\
\hline 合规性 & $\begin{array}{c}\text { 《教育部关于国家精品开放课 } \\
\text { 程建设的实施意见》 }\end{array}$ & $\begin{array}{c}\text { 《著作权集体管 } \\
\text { 理条例》 }\end{array}$ & $\begin{array}{c}\text { 《知识共享协议 } \\
\text { (CC)》 }\end{array}$ & 无 \\
\hline 开放共享性 & 一般 & 一般 & 高 & 高 \\
\hline 时间成本 & 中 & 一般 & 中 & 中 \\
\hline 经济成本 & 中 & 低 & 低 \\
\hline 普遍性 & 高 & 一般 & 极少 & 极少 \\
\hline
\end{tabular}

国内研究 MOOC 版权归属有三种说法，第一种是“讲授权利说”，是指权利归属于主要授 课人, 不包括辅助课程开展人员。第二种是“职务作品说”, 是指权利归属于参与 MOOC 制作 的所有职务人员。第三种“折中权衡说”是均衡“讲授权利说”和“职务作品说”、依具体情况通 过协议而定。[12]

表 4 是对国内研究 MOOC 版权归属的三种说法从权利归属人、成立理由、优点和缺点、 案例进行的对比, “折中权衡说” 是根据自治原则, 由当事双方事先以合同方式约定版权归属, 通过法律协议形式解决争议, 是目前普遍采用一种方式, “讲授权利说”因其权利归属人只有 主要授课人, 对参与 MOOC 制作的人员和学校及机构相对有欠公允, 在国内暂未找到相关案 例。

表4 2013年一2018年MOOC版权权利归属三种说法对比分析

\begin{tabular}{|l|c|c|c|}
\hline 主要特点 & 归属说法 & 授权利说 & 职务作品说 \\
\hline 权利归属人 & 主要授课人 & 资料制作者 & 妌授人与制作者共有 \\
\hline 成立理由 & $\begin{array}{c}\text { MOOC是基于授课人现场 } \\
\text { 演说且必须课人参与完成 }\end{array}$ & $\begin{array}{c}\text { MOOC属于职务作业, 是集 } \\
\text { 体共同权利 }\end{array}$ & 通过合同协议 \\
\hline 优点 & 肯定主要授课人的作用性 & $\begin{array}{c}\text { 肯定MOOC集体创作的现 } \\
\text { 实流程 }\end{array}$ & 较强的适应性 \\
\hline 缺点 & 忽略其他成员的贡献 & $\begin{array}{c}\text { 混淆了主体与局部的分属 } \\
\text { 关系, 易造成版权所有梳的 }\end{array}$ & $\begin{array}{c}\text { 需要依不同情况制定不同 } \\
\text { 协议, 时间成本较高 }\end{array}$ \\
\hline
\end{tabular}




\begin{tabular}{|l|c|c|c|}
\hline & 纠纷 & \\
\hline & & & 学堂在线、中国大学 \\
& MOOC、武汉大学与爱立信 \\
案例 & (中国) 通信有限公司合作 \\
& 美国AOL & $\begin{array}{c}\text { 南理工大学建设学院、延 } \\
\text { 边学、TED组织的欧洲分 } \\
\text { 部 }\end{array}$ & $\begin{array}{c}\text { 新闻网与英国牛津大学合 } \\
\text { 作的“欧陆文化公开课” }\end{array}$ \\
\hline
\end{tabular}

\section{5 图书馆承担 MOOC 版权支持服务的内容}

MOOC 版权有关的内容是广泛的, 专业性要求高, 由作为 MOOC 责任主体的某一课程 教师全权负责版权相关工作, 花费时间不但长, 且易有侵权的风险, 因此国内外很多大学图 书馆已经承担起 MOOC 版权支持服务。表 5 整理了图书馆承担 MOOC 版权支持服务的内容 及案例。

表5 图书馆承担MOOC版权支持的服务内容

\begin{tabular}{|c|c|c|c|}
\hline 序号 & 服务内容 & 版权清理内容 & 案例 \\
\hline 1 & 设立版权图书馆员岗位 & \multirow{8}{*}{$\begin{array}{c}\text { 和制作MOOC相关的所有教材、 } \\
\text { 文章、图片、数据、录音、录像、 } \\
\text { 教辅 }\end{array}$} & \multirow{8}{*}{ 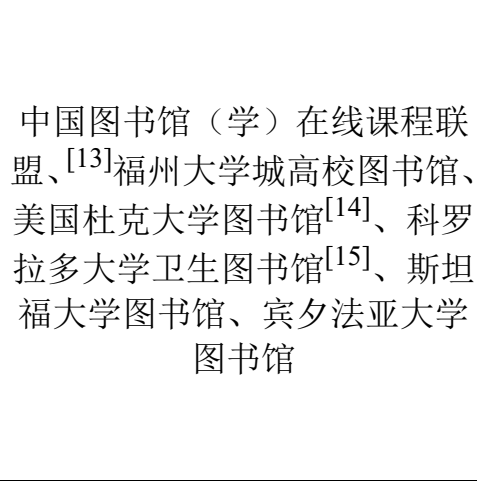 } \\
\hline 2 & 建立版权指南 & & \\
\hline 3 & 研究各国著作法相关法律 & & \\
\hline 4 & 合理使用的指导及咨询服务 & & \\
\hline 5 & 与出版商协商, 获取版权许可 & & \\
\hline 6 & 寻找和收集开放获取资源 & & \\
\hline 7 & 对教师进行版权培训 & & \\
\hline 8 & $\begin{array}{c}\text { 协助教师进行MOOC内容版权清 } \\
\text { 理 }\end{array}$ & & \\
\hline
\end{tabular}

\section{MOOC 版权建设与完善建议}

因国内 MOOC 的起步比较晚, 研究 MOOC 版权的文献较少, 其 MOOC 版权的相关问题 正在不断摸索和完善, 责权利还不清晰, 很多做法并无正式法律法规支持, 需要政府、学校、 教师各方协同发力，就此提出三个方面的建议:

一是对于政府来说, 需要组织专家就 MOOC 版权有关问题进行专题研究, 尽快出台相关 细则，明确平台方、教师团队、学校等各方版权责权利，规范 MOOC 建设应用。

二是对于学校来说, 应该主动作为, 可以考虑依托专业机构如图书馆, 组织对教师进行 相关的版权知识培训, 清除学校教师参与 MOOC 的版权障碍, 包括完善与教师、平台、出版 商之间的协议，明确课程拥有权和使用权，指导教师做好版权清理工作等。

三是对于教师来说, 要主动参加 MOOC 版权有关培训, 积极了解有关制度规范, 在制作 MOOC 过程中, 要高度重视和细致了解第三方材料的版权要求, 要有版权意识, 主动与学校、 课程承载平台明确责权利有关内容, 在遵从版权约定的前提下保护自己的合法权益。

\section{5. 结束语}

MOOC 的版权涵盖内容广，涉及的相关利益者多，相对于其它的出版物而言，版权问题 更加重要。现有的研究和实践为后续开展 MOOC 建设应用提供了版权归属、保护等积极借鉴, 但随着 MOOC 在校内教学和在线教育中的进一步应用, MOOC 版权建设将进一步规范和制 度化明确。 


\section{致谢}

本文为军事职业教育互联网服务平台阶段性成果之一。

\section{References}

[1] Hao Dan, Analysis of MOOC research literature status in China, Distance Education in China, vol.11, pp. 42-50, 2013.

[2] Information on Official website of Ministry of Education, $h t t p: / / w w w . m o e . g o v . c n / j y b \_x w f b / x w \_f b h / m o e \_2069 / x w f b h \_2018 n / x w f b \_20180115 / w z s l /$

[3] General Administration of Press and Publication, Regulations on the implementation of copyright law, http://www.law-lib.com/law/law_view.asp?id=410604

[4] General Administration of Press and Publication, The Berne Convention for the protection of literary and artistic works, http://www.law-lib.com/law/law_view.asp?id=98034

[5] Copyright law of the United States. 102nd

[6] Deng Peizhen, Study on Copyright Issues of Digital Resources in Library's Information Commons, Information Research, vol.05, pp. 15-21, 2017.

[7] Information on Official website of Ministry of Education, http://old.moe.gov.cn/publicfiles/business/htmlfiles/moe/s3843/201111/xxgk_126346.html

[8] Information on Baidu Encyclopedia, https://baike.baidu.com/item/Collective Management of Copyright Regulations

[9] Information on Baidu Encyclopedia, https://baike.baidu.com/item/creative\%20commons

[10]Wang Xiaoping, Study on the Copyright Issues in the Construction and Management of China's MOOC, The Library Journal of Shandong, vol.06, pp. 5-8, 2015.

[11]Fang Zhangwei, Comparison and legal regulation between MOOC and traditional classroom teaching from the perspective of copyright, View on Publishing, vol.10, pp. 79-81, 2016.

[12]Liu Jian, Analysis of copyright ownership in MOOCs courses in Colleges and Universities, Publishing Research, vol.11, pp. 88-91,2015.

[13] Information on Official website of Ministry of Education, http://www.moe.gov.cn/s78/A08/moe_745/201703/t20170302_297912.html

[14]FLOWER L,SMITH K, Drawing the Blueprint As We Build: Setting Up a Library-based Copyright and Permissions Service for MOOCs, http://www.dlib.org/dlib/july13/fowler/07fowler.html

[15]CU Health Sciences Library, CU MOOC copyright guidelines, http://hslibraryguides.ucdenver.edu/mooccoprightguidelines\#_ednref3 